Conclusion We found no evidence of a protective effect of hard water against acute MI admission, contrary to other published studies. This may be because our methods allowed us to better account for possible confounding variables. We go on to hypothesise that as water supply networks developed historically, with soft water from upland areas being used supply the needs of rapidly industrialising areas and other areas left to use local groundwater, some interesting accidental correlations between neighbourhood socio-economic status and water hardness arose which may have confounded previous work. However, mass differential exposure to highly bio-available minerals, especially calcium and magnesium, does raise some interesting further questions about the relationship between water supply and population health, which our methods can be used to investigate further.

\section{PSO9 SLEEP PROBLEMS: AN EMERGING GLOBAL EPIDEMIC? FINDINGS FROM THE INDEPTH WHO-SAGE STUDY AMONG OVER 40,000 OLDER ADULTS FROM EIGHT COUNTRIES ACROSS AFRICA AND ASIA}

doi:10.1136/jech-2012-201753.108

'S Stranges, 'W Tigbe, ${ }^{2} \mathrm{FX}$ Gómez-Olivé, 1'2M Thorogood, 'N-B Kandala. 'Division of Health Sciences, University of Warwick Medical School, Coventry, UK; ${ }^{2}$ MRC/Wits Rural Public Health \& Health Transitions Research Unit, University of the Witwatersrand, Agincourt, South Africa

Background Several studies have reported downward trends in the average duration of sleep and an increasingly higher prevalence of sleep problems across different Western populations. However, the evidence from low-income countries is limited. This study aims to fill this gap by examining the prevalence of sleep problems and associated factors in low-income settings.

Methods Community-wide samples were taken from eight countries across Africa and Asia participating in the INDEPTH WHOSAGE multicentre collaboration during 2006-2007. The participating sites included rural populations in Ghana, Tanzania, South Africa, India, Bangladesh, Vietnam and Indonesia, and an urban area in Kenya. The overall sample comprised 24,434 women and 19,501 men, for a total of 43,935 participants, aged 50 years and over. Two measures of sleep quality, over the last 30 days, were assessed alongside a number of socio-demographic variables, measures of quality of life, and co-morbidities.

Results Overall $16.6 \%$ of participants reported severe/extreme nocturnal sleep problems, with a striking variation across the eight populations, ranging from 3.9\% (Purworejo, Indonesia, and Nairobi, Kenya) to over $40.0 \%$ (Matlab, Bangladesh). There was a consistent pattern of higher prevalence of sleep problems in women and older age groups. In bivariate analyses, lower education, not living in partnership, and poorer self-rated quality of life were consistently associated with higher prevalence of sleep problems $(\mathrm{P}<0.001)$. In multivariate logistic regression analyses, limited physical functionality or greater disability and feelings of depression and anxiety were consistently strong, independent correlates of sleep problems, both in women and men, across the eight sites $(\mathrm{P}<0.001)$.

Conclusion A large number of older adults in low-income settings are currently experiencing sleep problems, which emphasises the global dimension of this emerging public health issue. This study corroborates the multifaceted nature of sleep problems, which are strongly linked to poorer general wellbeing and quality of life, and psychiatric co-morbidities.

\section{PS10 THE APPROPRIATENESS OF, AND COMPLIANCE WITH, TELEPHONE TRIAGE DECISIONS}

doi:10.1136/jech-2012-201753.109

L Blank, J Coster, A Cathain, E Knowles, J Tosh, J Turner, J Nicholl. ScHARR, University of Sheffield, Sheffield, UK
Background Telephone triage plays an important role in managing demand for healthcare. In recent years there has been an increasing use of telephone triage in managing demand for unscheduled health care for general health problems. Policy makers, service providers and service evaluators are interested in the appropriateness of triage decisions in the context of offering an effective and safe service. In 2009 the Department of Health in England announced the establishment of a small number of pilot sites to test a new telephone triage service called 'NHS 111'. The service offers telephone triage to members of the general population calling about urgent but nonemergency health problems. Calls are triaged by trained non-clinical call advisors and directed to a range of services such as an emergency department, or a general practitioner.

Methods As part of an evaluation of these pilots, in order to provide context for understanding the findings, a rapid evidence assessment was undertaken of the appropriateness of, and compliance with, telephone triage to synthesise the evidence on the percentage of telephone triage decisions assessed as appropriate and the percentage which callers complied with. The aim of this paper is to report on a systematic review of the literature on appropriateness of, and compliance with, telephone triage decisions. The study focused on telephone triage services directing patients to an appropriate health care provider. The principles of rapid evidence assessment were followed.

Results We identified 54 relevant papers; 26 papers reported appropriateness of triage decision, 26 papers reported compliance with triage decision and 2 papers reported both. Nurses triaged calls in most of the studies $(n=49)$. Triage decisions rated as appropriate varied between $44 \%$ and $98 \%$ (median 75 ); compliance ranged from $56 \%$ to $98 \%$ (median $77 \%$ ). Variation could not be explained by type of service or method of assessing appropriateness. However, inconsistent definitions of appropriateness may explain some variation. Triage decisions to contact primary care (median 66\%, range 25\%91\%) may have lower compliance than decisions to contact emergency services (median $75 \%$, range $29 \%-100 \%$ ) or self care (median $77 \%$, range $26 \%-100 \%$ )

Conclusion Telephone triage services can offer appropriate decisions, and decisions that callers comply with. However the association between the appropriateness of a decision and subsequent compliance requires further investigation and further consideration needs to be given to the minority of calls which are inappropriately managed. We suggest that a definition of appropriateness incorporating both accuracy and adequacy of triage decision should be encouraged.

\section{PS11 DOES CONTACT WITH A PODIATRIST PREVENT THE OCCURRENCE OF A LOWER EXTREMITY AMPUTATION IN PEOPLE WITH DIABETES? A SYSTEMATIC REVIEW AND META-ANALYSIS}

doi:10.1136/jech-2012-201753.110

${ }^{1} \mathrm{CM}$ Buckley, ${ }^{2} \mathrm{PM}$ Kearney, ${ }^{2} \mathrm{JJ}$ Perry, ${ }^{1} \mathrm{CP}$ Bradley. ${ }^{1}$ Department of GP, UCC, Cork, Ireland; ${ }^{2}$ Department of Epidemiology and Public Health, UCC, Cork, Ireland

Background Diabetes is associated with a significant risk of LEA (lower extremity amputation). LEA rates vary between communities, 46-9,600 per $10^{5}$ people with diabetes, for many reasons. The effects of clinical and socio-demographic risk factors on the occurrence of a LEA have been well documented in people with diabetes. However, the effect of patient contact with a podiatrist on the prevention of LEA in people with diabetes is less well explored. The objective of this study was to determine if contact with a podiatrist prevents the occurrence of lower extremity amputation in people with diabetes.

Methods We conducted a systematic review of available published literature on the effect of contact with a podiatrist on the prevention of lower extremity amputation in people with diabetes. Eligible 